\title{
Search for Neutrino Oscillations at the Palo Verde Nuclear Reactors
}

\author{
F. Boehm, ${ }^{3}$ J. Busenitz, ${ }^{1}$ B. Cook,${ }^{3}$ G. Gratta, ${ }^{4}$ H. Henrikson, ${ }^{3}$ J. Kornis, ${ }^{1}$ D. Lawrence, ${ }^{2}$ K. B. Lee, ${ }^{3}$ K. McKinny, \\ L. Miller, ${ }^{4}$ V. Novikov, ${ }^{3}$ A. Piepke, ${ }^{1,3}$ B. Ritchie, ${ }^{2}$ D. Tracy, ${ }^{4}$ P. Vogel, ${ }^{3}$ Y-F. Wang, ${ }^{4}$ and J. Wolf ${ }^{1}$ \\ ${ }^{1}$ Department of Physics and Astronomy, University of Alabama, Tuscaloosa, Alabama 35487 \\ ${ }^{2}$ Department of Physics and Astronomy, Arizona State University, Tempe, Arizona 85287 \\ ${ }^{3}$ Division of Physics, Mathematics and Astronomy, Caltech, Pasadena, California 91125 \\ ${ }^{4}$ Physics Department, Stanford University, Stanford, California 94305
}

(Received 22 December 1999)

\begin{abstract}
We report on the initial results from a measurement of the antineutrino flux and spectrum at a distance of about $800 \mathrm{~m}$ from the three reactors of the Palo Verde Nuclear Generating Station using a segmented gadolinium-loaded scintillation detector. We find that the antineutrino flux agrees with that predicted in the absence of oscillations excluding at 90\% C.L. $\bar{\nu}_{e}-\bar{\nu}_{x}$ oscillations with $\Delta m^{2}>1.12 \times 10^{-3} \mathrm{eV}^{2}$ for maximal mixing and $\sin ^{2} 2 \theta>0.21$ for large $\Delta m^{2}$. Our results support the conclusion that the atmospheric neutrino oscillations observed by Super-Kamiokande do not involve $\nu_{e}$.
\end{abstract}

PACS numbers: 14.60.Pq, 13.15.+g, 14.60.Lm, 25.30.Pt

Nuclear reactors have been used as intense sources of $\bar{\nu}_{e}$ in experiments searching for neutrino oscillations [1]. These experiments usually detect $\bar{\nu}_{e}$ by the process $\bar{\nu}_{e}+$ $p \rightarrow n+e^{+}$, where the cross-section-weighted energy spectrum of $\bar{\nu}_{e}$, peaking at about $4 \mathrm{MeV}$, can be deduced from the measured $e^{+}$spectrum. Any $\bar{\nu}_{e}$ flux deficit or distortions of the $\bar{\nu}_{e}$ energy spectrum would indicate oscillations. The low energy of reactor $\bar{\nu}_{e}$ allows these experiments to reach very small mass parameters, albeit with modest mixing-angle sensitivity. Past experiments [2] with detectors at $50-100 \mathrm{~m}$ from a reactor have explored the mass-parameter range down to $10^{-2} \mathrm{eV}^{2}$. The work described here and a similar experiment elsewhere [3] are the first long baseline $(\sim 1 \mathrm{~km})$ searches, designed to explore the parameter range down to $10^{-3} \mathrm{eV}^{2}$ as suggested by the early Kamiokande atmospheric neutrino anomaly [4]. Although later results from Super-Kamiokande [5] (appeared while this work was in progress) seem to disfavor the $\nu_{\mu}-\nu_{e}$ channel, a direct experimental exploration amply motivated this work.

The Palo Verde neutrino oscillation experiment is located at the Palo Verde Nuclear Generating Station near Phoenix, Arizona. The total thermal power from three identical pressurized water reactors is $11.6 \mathrm{GW}$. Two of the reactors are $890 \mathrm{~m}$ from the detector, while the third is at $750 \mathrm{~m}$. Our detector is placed in a shallow underground site (32-m-water-equivalent overburden), thus eliminating the hadronic component of cosmic radiation and reducing the muon flux by a factor of $\sim 5$. The fiducial mass, segmented to reject the remaining background, consists of 11.3 tons of $0.1 \%$ Gd-loaded liquid scintillator contained in a $6 \times 11$ array of 9-m-long acrylic cells, as shown in Fig. 1. Each cell is viewed by two 5-in. photomultiplier tubes, one at each end. A $\bar{\nu}_{e}$ is identified by space- and time-correlated $e^{+}$and $n$ signals. Positrons deposit their energies in the scintillator and annihilate, yielding two 511-keV $\gamma$ 's, giving a triple coincidence. Neutrons ther- malize and are captured in Gd, giving a $\gamma$-ray shower of $8 \mathrm{MeV}$ total energy.

The Gd loading of the scintillator has two advantages: it reduces the neutron capture time from 170 (on protons) to $30 \mu s$ and provides a high energy $\gamma$ shower to tag the neutron capture, resulting in a substantial background reduction. Both the positron and the neutron are triggered by a triple coincidence requiring at least one cell above a "high" threshold set at about $600 \mathrm{keV}$ (positron ionization or neutron shower core) and two cells above a "low" threshold set at about $40 \mathrm{keV}$ (Compton scattering from annihilation photons or neutron shower tails). The triple coincidences are required to be within a $3 \times 5$ matrix anywhere in the detector.

The central detector is surrounded by a 1-m water shield to moderate background neutrons produced by muons outside the detector and to absorb $\gamma$ 's from the laboratory

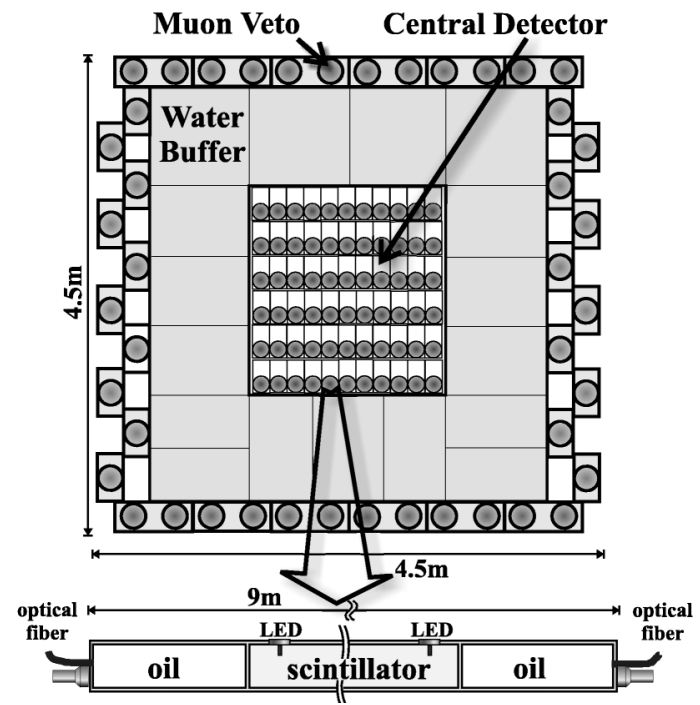

FIG. 1. Schematic view of the Palo Verde neutrino detector. 
walls. Outside the water tanks are 32 large liquid scintillator counters and two end caps to veto cosmic muons. The rate of cosmic muons is approximately $2 \mathrm{kHz}$. The pattern of muons traveling through veto chambers and their timing relative to the central detector hits are recorded for subsequent off-line analysis. The central detector is equipped with a system of tubes that allows the insertion of calibration sources in the small spaces between cells. In addition, a set of blue light-emitting diodes (LED's) and optical fibers can produce flashes of light inside each of the cells. In order to reduce natural radioactivity, all building materials for the detector are carefully selected, including the aggregate (marble) used in the concrete of the underground laboratory.

The detector geometry, materials, and electromagnetic interactions are simulated using the package GEANT. Hadronic interactions are described by GFLUKA and the low energy neutron transport is simulated by GCALOR. The inclusive gamma spectrum from neutron capture on Gd is specially modeled according to measurements [6]. Light quenching effects are also included [7].

Since the ultimate sensitivity of the experiment relies on a disappearance measurement, precise knowledge of the detector efficiency and of the expected $\bar{\nu}_{e}$ flux from the reactors is essential.

The efficiency calibration is based upon a primary measurement performed a few times per year with a calibrated ${ }^{22} \mathrm{Na} e^{+}$source and an Am-Be neutron source. The ${ }^{22} \mathrm{Na}$ source is placed in the calibration pipes and mimics the effects of the positron from the $\bar{\nu}_{e}$ interaction by providing annihilation radiation and a $1.275-\mathrm{MeV}$ photon which simulates the $e^{+}$ionization in the scintillator. The source is placed at 18 positions in the detector deemed to be representative of different conditions. The neutron detection efficiency is measured by scanning the detector with the Am-Be source where the 4.4-MeV $\gamma$ associated with the neutron emission is tagged with a miniaturized $\mathrm{NaI}(\mathrm{Tl})$ counter.

Other calibrations, used to measure the detector energy response, are performed using the Compton edges from ${ }^{137} \mathrm{Cs},{ }^{65} \mathrm{Zn}$, and ${ }^{228} \mathrm{Th}$ sources. The same Th source is also used more frequently to track the scintillator transparency. Weekly runs of the fiber-optic and LED flasher systems are used, respectively, to monitor the gain and linearity of photomultipliers and the timing/position relationship along the cells.

Since the energy deposition of the 511-keV $\gamma$ 's in one cell has a sharp falling spectrum (Compton scattering) it is vital to have the lowest possible low thresholds in the trigger and to understand the behavior of such thresholds with great accuracy. This second task is complicated by the fact that the trigger uses voltage amplitudes, while only charge from integrating analog-to-digital converters is available off-line. For this reason our detector simulation includes a detailed description of the signal development in time. This code correctly describes the shape of pulses taking into account scintillator light yield, attenuation length, and deexcitation time; photomultiplier rise and fall time and gain; and event position along a cell. The simulation of the detector response to the ${ }^{22} \mathrm{Na}$ source correctly describes the $40-\mathrm{keV}(600-\mathrm{keV})$ threshold position to within $1.4 \mathrm{keV}$ $(2.6 \mathrm{keV})$, resulting in an uncertainty on the positron (neutron) efficiency of $4 \%(3 \%)$.

The $\bar{\nu}_{e}$ flux and spectrum from a fission reactor and the $\bar{\nu}_{e}+p \rightarrow n+e^{+}$cross section are well known $[1,2,8]$ and are calculated by tracking the ${ }^{235} \mathrm{U},{ }^{238} \mathrm{U},{ }^{239} \mathrm{Pu}$, and ${ }^{241} \mathrm{Pu}$ fission rates in the three plant reactors, taking into account both power level and fuel age. The uncertainty in the $\bar{\nu}_{e}$ reaction rate is less than $3 \%$.

The data presented here were collected in periods of 67.3 days in 1998 and 134.4 days in 1999. During the 1998 (1999) data taking one of the far (near) reactors was off for 31.3 (23.4) days. While a detailed description of the data analysis will be reported elsewhere [9], here we will outline the principles of the analysis and the results.

Neutrino candidates were selected by requiring an appropriate pattern of energy to be present in the detector for the positronlike and the neutronlike parts of the events. In addition the two subevents are required to occur closer than about $1 \mathrm{~m}$ from each other.

At our depth the background to $\bar{\nu}_{e}$ events consists of two types of events: uncorrelated hits from cosmic rays and natural radioactivity and correlated ones from cosmicmuon-induced neutrons. The first type can be measured by studying the time difference between positronlike and neutronlike parts of an event. By requiring that the time lapse between the two subevents $t_{\mathrm{en}}$ be $5<t_{\mathrm{en}}<200 \mu \mathrm{s}$, the uncorrelated background is reduced to $3.4 \pm 0.2$ events $\mathrm{d}^{-1}\left(4.8 \pm 0.2\right.$ events $\left.\mathrm{d}^{-1}\right)$ for 1998 (1999), as measured from a fit to an appropriate combination of exponential functions.

The distribution of time intervals between a cosmic-ray $\mu$ crossing the detector and a $\bar{\nu}_{e}$-like event carries information on the correlated background. From an exponential fit we infer that the majority of correlated background is produced by pairs of neutrons, where the capture of the first neutron in each pair mimics the positron signature. The requirement that no cosmic-ray hits be present in a window of $150 \mu \mathrm{s}$ preceding the $\bar{\nu}_{e}$ candidate completes the event selection. While the detector efficiency, $\eta$, is dependent upon the neutrino energy, the efficiency integrated over the neutrino spectrum produced by the reactor (i.e., in the case of no oscillation) is $\simeq 8 \%(\simeq 11 \%)$ for 1998 (1999) (the higher efficiency in 1999 is due to improvements in the data acquisition dead time and trigger efficiency).

The resulting rates $N$ of $\bar{\nu}_{e}$ candidates per day in different periods are given in Table I. Along with the $\bar{\nu}_{e}$ events this final data set contains the random background mentioned above and a substantial amount of correlated background. Two independent techniques were used to estimate and subtract the background. The most straightforward method ("Method 1") relies on the changes 
TABLE I. Summary of results from the Palo Verde experiment. The values in the second part of the table are derived from Method 2. $B=B_{\text {unc }}+B_{n n}+B_{n p} . \quad R_{\text {Obs }}$ and $R_{\text {Calc }}$ are the observed and calculated $\bar{\nu}_{e}$ rates corrected by the efficiencies $\eta$ for the case of no oscillations. *Reactor at $890 \mathrm{~m}$ distance off. ${ }^{\dagger}$ Reactor at $750 \mathrm{~m}$ distance off. Statistical uncertainties only.

\begin{tabular}{lcccc}
\hline \multicolumn{1}{c}{ Period } & 1998 “on" & 1998 “off”* & 1999 “on" & 1999 “off” " \\
\hline Duration $(\mathrm{d})$ & 36.0 & 31.3 & 111.0 & 23.4 \\
$\eta$ & 0.0746 & 0.0772 & 0.112 & 0.111 \\
$N\left(\mathrm{~d}^{-1}\right)$ & $38.2 \pm 1.0$ & $32.2 \pm 1.0$ & $52.9 \pm 0.7$ & $43.9 \pm 1.4$ \\
\hline$S_{\nu}\left(\mathrm{d}^{-1}\right)$ & $16.5 \pm 1.4$ & $13.4 \pm 1.4$ & $25.2 \pm 0.9$ & $15.1 \pm 1.9$ \\
$B\left(\mathrm{~d}^{-1}\right)$ & $21.7 \pm 1.0$ & $18.8 \pm 1.0$ & $27.7 \pm 0.6$ & $28.8 \pm 1.3$ \\
$R_{\text {Obs }}\left(\mathrm{d}^{-1}\right)$ & $221 \pm 18$ & $174 \pm 17$ & $225 \pm 8$ & $136 \pm 17$ \\
$R_{\text {Calc }}\left(\mathrm{d}^{-1}\right)$ & 218 & 155 & 218 & 130 \\
\hline \hline
\end{tabular}

of the $\bar{\nu}_{e}$ signal when different reactors are turned off. For simplicity we first directly subtract the efficiencycorrected rates $r=N / \eta$ for "off" periods from the "on" periods. For 1998 (1999) we compare the efficiencycorrected rates of observed interactions $95.0 \pm 18.6 \mathrm{~d}^{-1}$ $\left(76.8 \pm 13.6 \mathrm{~d}^{-1}\right)$ with the prediction of $63 \mathrm{~d}^{-1}\left(88 \mathrm{~d}^{-1}\right)$. While the uncertainties quoted here are statistical only, we find good agreement with the hypothesis of no oscillations. We can then proceed to add the 1998 and 1999 data sets (which have a different combination of baselines) and obtain a neutrino energy spectrum for the difference "on"-"off." This spectrum well matches the model for no oscillations as shown in Fig. 2.

In order to test quantitatively the oscillation hypothesis in the $\Delta m^{2}-\sin ^{2} 2 \theta$ plane we perform a $\chi^{2}$ analysis comparing the expected to the measured rates. Information on the reactor neutrino flux, in the form of burnup dependent fission rates, is computed for every detector run (on average $\sim 12 \mathrm{~h}$ of data). This analysis effectively unfolds the background from the data using the $\bar{\nu}_{e}$-flux variation due to reactor power changes and fuel burnup. At each point of the $\Delta m^{2}-\sin ^{2} 2 \theta$ grid the energy spectrum and the detector efficiency are calculated and used to predict the expected rates $\rho_{i}$, where $i$ denotes the different detector runs. We then construct the function

$$
\chi^{2}=\sum_{i} \frac{\left[\left(\alpha \rho_{i}+b\right)-N_{i}\right]^{2}}{\sigma_{i}^{2}}+\frac{(\alpha-1)^{2}}{\sigma_{\text {syst }}^{2}} .
$$

The $\chi^{2}$ is minimized with respect to the background contribution $b$ to the candidate neutrino rate $N_{i}$ and the parameter $\alpha$ that accounts for possible global normalization effects. While statistical errors $\sigma_{i}$ have to be individually applied to each run, the systematic error $\sigma_{\text {syst }}$ is treated as a global parameter in the $\chi^{2}$. The contribution of different sources of systematics to $\sigma_{\text {syst }}$ is given in the first column in Table II. The effect of the $\bar{\nu}_{e}$ selection cuts is estimated by a random sampling of the unity hypervolume defined by conservative ranges for each of the individual cuts. This technique properly takes into account the possible correlations between cuts. The $90 \%$

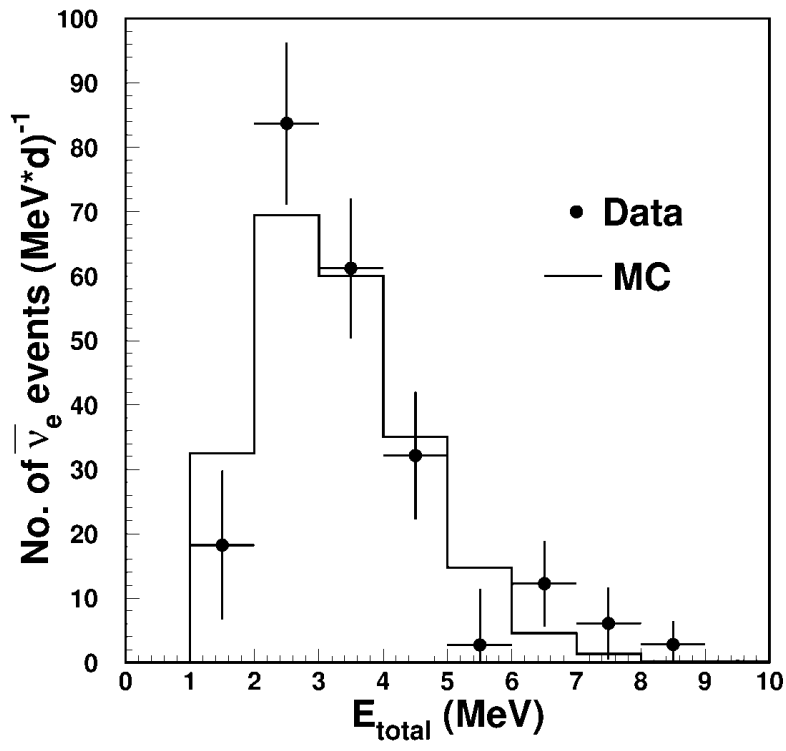

FIG. 2. Spectrum of energy deposited for neutrino events after "on"-"off" background subtraction. The sum of the 1998 and 1999 distributions is compared with the Monte Carlo simulation assuming no oscillations. Uncertainties are statistical only. The $\chi^{2} /$ n.d.f. between simulation and data is $8.5 / 8$, supporting the no-oscillation scenario.

C.L. acceptance region is defined in accordance with the procedure in [10] by $\Delta \chi^{2}>\chi^{2}\left(\Delta m^{2}, \sin ^{2} 2 \theta\right)-\chi_{\text {best }}^{2}$, where $\chi^{2}\left(\Delta m^{2}, \sin ^{2} 2 \theta\right)$ describes the fit quality at the current grid point and $\chi_{\text {best }}^{2}$ describes that of the global best fit determined in the physically allowed parameter space. This procedure clearly prefers a no-oscillation scenario as illustrated in Fig. 3 (curve $a$ ). The fit also provides an estimate of the background $b=19.5 \pm$ $1.3 \mathrm{~d}^{-1}\left(b=26.3 \pm 1.7 \mathrm{~d}^{-1}\right)$ for 1998 (1999), and of $\alpha=1.02 \pm 0.08$ with a $\chi^{2}$ per number of degrees of freedom $\chi^{2} /$ n.d.f. $=318 / 327$.

In an independent analysis ("Method 2") that is described in detail elsewhere [11] we make use of the intrinsic symmetry of the dominant two-neutron background to cancel most of the background directly from data and compute the remaining components from Monte Carlo simulations. This technique makes the best possible use of the statistical power of all data collected. The rate of candidate events after all cuts can be written as $N=B_{\text {unc }}+B_{n n}+B_{p n}+S_{\nu}$, where the contribution of the uncorrelated $B_{\text {unc }}$, two-neutron $B_{n n}$, and other correlated backgrounds $B_{p n}$ are explicitly represented, along with the $\bar{\nu}_{e}$ signal $S_{\nu}$. The dominant background $B_{n n}$ (along with $B_{\text {unc }}$ ) is symmetric under exchange of subevents, so that an event selection with the requirements for the prompt and delayed event parts swapped will result in a rate $N^{\prime}=B_{\text {unc }}+B_{n n}+\epsilon_{1} B_{p n}+\epsilon_{2} S_{\nu}$, where $\epsilon_{1}$ and $\epsilon_{2}$ account for the different efficiency for selecting asymmetric events after the swap. We then calculate $N-N^{\prime}=\left(1-\epsilon_{1}\right) B_{p n}+\left(1-\epsilon_{2}\right) S_{\nu}$, where the efficiency correction $\epsilon_{2} \simeq 0.2$ can be estimated from 
TABLE II. Origin and magnitude of systematic errors. Using Method 2 for background subtraction reduces the systematic uncertainty from the event selection cuts but introduces a new uncertainty due to the accuracy of the Monte Carlo used for the estimate of $B_{p n}$.

\begin{tabular}{lcc}
\hline \hline \multicolumn{1}{c}{ Systematic } & Method 1 $(\%)$ & Method 2(\%) \\
\hline$e^{+}$efficiency & 4 & 4 \\
$n$ efficiency & 3 & 3 \\
$\bar{\nu}_{e}$ flux prediction & 3 & 3 \\
$\bar{\nu}_{e}$ selection cuts & 8 & 4 \\
$B_{p n}$ estimate & $\ldots$ & 4 \\
\hline \multicolumn{1}{c}{ Total } & 10 & 8 \\
\hline \hline
\end{tabular}

the $\bar{\nu}_{e}$ Monte Carlo simulation. We find that the processes of $\mu$ spallation in the laboratory walls and capture of the $\mu$ 's that are not tagged by the veto counter $(4 \pm 1) \%$ contribute to $\left(1-\epsilon_{1}\right) B_{p n}$, while other backgrounds are negligible. Using Monte Carlo simulation, we obtain $\left(1-\epsilon_{1}\right) B_{p n}=-0.9 \pm 0.5 \mathrm{~d}^{-1}\left(-1.3 \pm 0.6 \mathrm{~d}^{-1}\right)$ for $\mu$ spallation in 1998 (1999); the same figures for $\mu$ capture are $0.6 \pm 0.3 \mathrm{~d}^{-1}\left(0.9 \pm 0.5 \mathrm{~d}^{-1}\right)$ in 1998 (1999). This represents only a small correction to $N-N^{\prime}$ since $\epsilon_{1}$ is close to 1 . While the Monte Carlo model is accurate for the capture process, in the case of spallation we simulate the broad range of spectral indexes for the $n$-recoil energy reported in literature [11]. The average between different predictions is then used for $B_{p n}$ while the spread is used as an extra systematic error in the second column in Table II. Since no $\bar{\nu}_{e}$ signal is present above $10 \mathrm{MeV}$, the observed integrated rate above such energy is used as a normalization of the Monte Carlo. The results are shown in the second part of Table I for different running periods. Clearly Method 2 is also in agreement with the no-oscillation hypothesis. The excluded region, calculated by comparing the expected and observed $\bar{\nu}_{e}$ rates taking into account the effect of the oscillation parameters on $\eta$ and $\epsilon_{2}$, is given in Fig. 3 (curve $b$ ).

In conclusion, the data from the first period of running from the Palo Verde detector shows no evidence for $\bar{\nu}_{e}-\bar{\nu}_{x}$ oscillations. This result, together with the data already reported by Super-Kamiokande [5] and a more stringent limit by Chooz [3], excludes the channel $\nu_{\mu}-\nu_{e}$ as being responsible for the atmospheric neutrino anomaly reported by Kamiokande [4]. Data taking at Palo Verde is scheduled to continue until the summer of 2000.

We thank the Arizona Public Service Company for the generous hospitality at the Palo Verde plant. The important contributions of M. Chen, R. Hertenberger, K. Lou, and $\mathrm{N}$. Mascarenhas in the early stages of this project are gratefully acknowledged. We are indebted to J. F. Beacom, B. Barish, R. Canny, A. Godber, J. Hanson, D. Michael, C. Peck, C. Roat, N. Tolich, and A. Vital for their help. We also acknowledge the generous financial help from

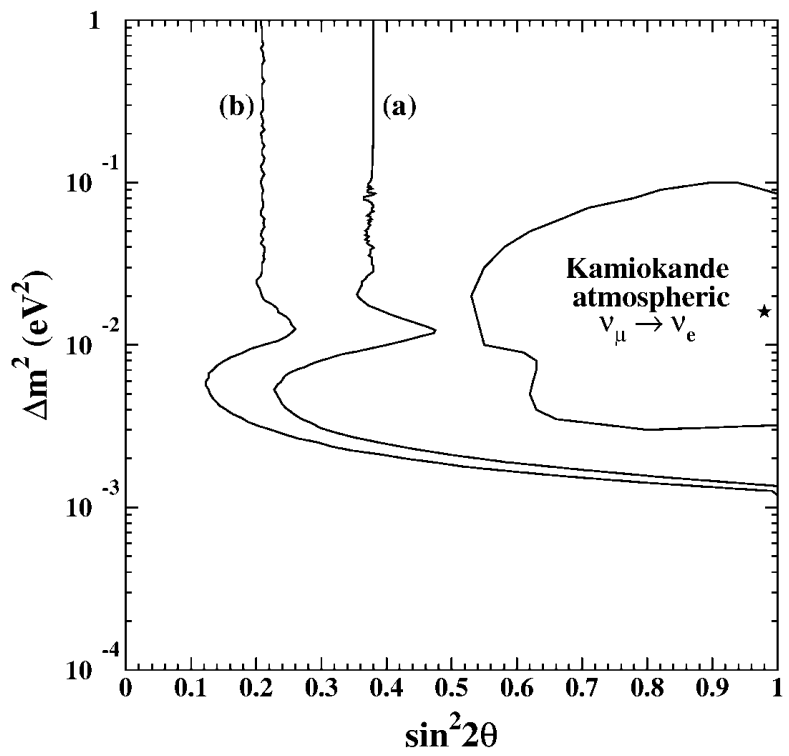

FIG. 3. Limits on mass difference and maxing angle from the present work (90\% C.L.). Curves $(a)$ and $(b)$ are based on Methods 1 and 2 for background subtraction, respectively, as described in the text. The method [10] is used to calculate the exclusion contours. The Kamiokande $\nu_{\mu}-\nu_{e}$ atmospheric neutrino result is also shown.

the University of Alabama, Arizona State University, Caltech, and Stanford University. Finally our gratitude goes to CERN, DESY, FNAL, LANL, LLNL, SLAC, and TJNAF which at different times provided us with parts and equipment for the experiment. This project was supported in part by the U.S. DoE. One of us (J. K.) received support from the Hungarian OTKA fund and another (L. M.) from the ARCS foundation.

[1] See, e.g., F. Boehm and P. Vogel, Physics of Massive Neutrinos (Cambridge University Press, Cambridge, England, 1992), 2nd ed.

[2] G. Zacek et al., Phys. Rev. D 34, 2621 (1986); B. Achkar et al., Nucl. Phys. B434, 503 (1995).

[3] M. Apollonio et al., Phys. Lett. B 420, 397 (1998); M. Apollonio et al., Phys. Lett. B 466, 415 (1999).

[4] Y. Fukuda et al., Phys. Lett. B 335, 237 (1994).

[5] Y. Fukuda et al., Phys. Rev. Lett. 81, 1562 (1998).

[6] L. V. Groshev et al., Nucl. Data Tables A5, 1 (1968).

[7] R. L. Craun and D. L. Smith, Nucl. Instrum. Methods 80, 239 (1970).

[8] P. Vogel and J. F. Beacom, Phys. Rev. D 60, 053003 (1999).

[9] F. Boehm et al. (to be published).

[10] G. J. Feldman and R. D. Cousins, Phys. Rev. D 57, 3873 (1998).

[11] Y.F. Wang et al., Phys. Rev. D (to be published) hep-ex/ 0002050 . 